\title{
The effects of 12-week psyllium fibre supplementation or healthy diet on blood pressure and arterial stiffness in overweight and obese individuals
}

\author{
Sebely Pal*, Alireza Khossousi, Colin Binns, Satvinder Dhaliwal and Simone Radavelli-Bagatini \\ School of Public Health, Curtin Health Innovation Research Institute, ATN Centre for Metabolic Fitness, Curtin University \\ of Technology, GPO Box U1987, Perth, WA 6845, Australia
}

(Received 10 December 2010 - Revised 25 May 2011 - Accepted 1 June 2011 - First published online 26 July 2011)

\section{Abstract}

Endothelial dysfunction and increased arterial stiffness occur early in the pathogenesis of the metabolic syndrome and they are both powerful independent predictors of cardiovascular risk. A high-fibre diet has been correlated with lower BMI and a lower incidence of hyperlipidaemia, CVD, hypertension and diabetes. The present randomised, parallel-design study compared the effects of fibre intake from a healthy diet $v$. fibre supplement diets on blood pressure (BP) and vascular function over 12 weeks. Overweight and obese adults were randomised to one of three groups: control (with placebo), fibre supplement (FIB) or healthy eating group with placebo (HLT). Systolic blood pressure (SBP) was lower in the FIB group compared with the control group at week 6, but not at week 12. However, SBP was lower in the HLT group compared with control group at week 12. At week 6, the FIB group presented lower diastolic blood pressure and augmentation index compared with the control group, but this result did not persist to the end of the study. The present study did not show any improvements in BP or vascular function in overweight and obese individuals with psyllium fibre supplementation over 12 weeks of intervention. However, a healthy diet provided the greatest improvements in BP in overweight and obese subjects. Further research with hypertensive individuals is necessary to elucidate whether increased fibre consumption in the form of psyllium supplementation may provide a safe and acceptable means to reduce BP, vascular function and the risk of developing CVD.

Key words: Fibre: Vascular function: Blood pressure: Obesity: CVD: Psyllium: Metabolic syndrome

Obesity is one of today's most blatantly visible public health problems, with an escalating global epidemic of overweight and obesity taking over many parts of the world ${ }^{(1)}$. Even in many low-income countries, obesity is now rapidly increasing, and often coexists in the same population with chronic undernutrition $^{(2)}$. Obesity significantly increases the risk of developing the metabolic syndrome, which carries with it the risk of developing CVD and type 2 diabetes mellitus. The metabolic syndrome consists of a combination of different abnormalities $^{(3)}$, such as abdominal obesity, atherogenic dyslipidaemia, insulin resistance, glucose intolerance, impaired endothelial function, elevated blood pressure (BP) and inflammation ${ }^{(4)}$. Endothelial dysfunction and hypertension are associated with increased cardiovascular (CVD) morbidity and mortality and have been implicated in the development of atherosclero$\mathrm{sis}^{(5)}$. A small reduction in $\mathrm{BP}$ of $2-5 \mathrm{mmHg}$ results in reductions in total mortality and mortality as a result of stroke and $\mathrm{CVD}^{(6)}$. It has been proposed that diet may play an important role in lowering $\mathrm{BP}$ and improving endothelial function ${ }^{(7-9)}$.
Epidemiological studies ${ }^{(10,11)}$ and clinical trials ${ }^{(12,13)}$ have shown that the intake of dietary fibre either in the form of food or supplements ${ }^{(14)}$ may be inversely correlated with BP levels and might be able to decrease the risk of hypertension development ${ }^{(15)}$. Accordingly, an increase in dietary fibres has been recommended by the WHO as a safe and practical approach to CVD risk reduction in the hypertensive population $^{(16)}$.

In an 8-week parallel-design study, treated hypertensive patients were randomised to one of four groups, consuming a low-protein (12.5\% energy), low-fibre $(15 \mathrm{~g} / \mathrm{d})$ diet alone or having supplements of soya protein ( $25 \%$ energy), psyllium (12 g fibre/d) or both protein and fibre ${ }^{(17)}$. Results of this study suggest that a diet containing fibre-rich grain, fruit and vegetables significantly decreases the need for anti-hypertension medication and improves BP control in individuals with hypertension $^{(17)}$.

Clinical trials on dietary fibre supplementation have shown a wide variation in BP responses. In trials with concentrated fibre supplements ${ }^{(13,18,19)}$, reductions in BP were larger

Abbreviations: AI, augmentation index; BP, blood pressure; DBP, diastolic blood pressure; FIB, fibre supplement group; HLT, healthy eating with placebo; SBP, systolic blood pressure.

*Corresponding author: Associate Professor S. Pal, fax +61 89266 2258, email s.pal@curtin.edu.au 
than those found in trials with fibre-rich foods. Furthermore, a meta-analysis on published randomised trials indicates that increased dietary fibre consumption (from 7 to $13.3 \mathrm{~g} / \mathrm{d}$ ), from mixed supplements pills, guava fruit or psyllium ${ }^{(13,17,20,21)}$, may provide a safe and acceptable means to reduce BP in patients with hypertension ${ }^{(18)}$ compared with those consuming $<7 \mathrm{~g} / \mathrm{d}$. The study by Eliasson et $a l^{(13)}$ showed that dietary fibre tablets containing a mixture of beet, barley and citrus in a ratio of 60:30:10 (7 g/d) significantly reduced diastolic blood pressure (DBP) in mildly hypertensive patients (from 18 to 70 years old) compared with a control group taking placebo ( $1 \mathrm{~g}$ fibre $(\mathrm{starch}) / \mathrm{d})$ tablets. These changes were independent of changes in body weight ${ }^{(13)}$. In the study by Burke et al. ${ }^{(17)}$, hypertensive patients were randomised to one of four groups in an 8-week factorial study of parallel design in which they either (1) followed a lowprotein, low-fibre diet, (2) had supplements of soya protein to increase protein intake to $25 \%$ energy, (3) had psyllium supplementation to provide an additional $12 \mathrm{~g}$ soluble fibre/d or (4) had both protein and fibre. Results showed that the combination of dietary protein and soluble fibre supplements lowered BP additively (group 4) in hypertensive individuals $^{(17)}$. In another study ${ }^{(21)}$, seventy-two hypertensive patients consuming a soluble diet containing $0.5-1.0 \mathrm{~kg}$ of guava daily (13.3 g fibre/d) for 4 weeks had a decreased systolic blood pressure (SBP) and DBP compared with those in the control group (consuming their usual diet). Another study has shown a significant reduction in both SBP and DBP in individuals (presenting with high BP) consuming a diet supplemented with $8 \mathrm{~g} / \mathrm{d}$ of water-soluble fibre from oat bran compared with those consuming a low-fibre diet $(1.3 \mathrm{~g} / \mathrm{d})^{(22)}$.

In animal models, soluble fibre supplementation has been suggested to improve the endothelial function and $\mathrm{BP}^{(23)}$ A diet supplemented with 3.5\% plantago ovata husks (psyllium) for 25 weeks has been shown to reduce SBP in obese diabetic Zucker rats ${ }^{(23)}$ as well as in spontaneously hypertensive rats supplemented a 3 and $10 \%$ diet for $30 \mathrm{~d}^{(24)}$. In addition, Goto Kakizaki type 2 diabetic rats presented reduced SBP following long-term consumption of cereal dietary fibre $(8 \mathrm{~g}$ barley/100 g of diet) for 16 weeks ${ }^{(25)}$. Both results suggest that a high-fibre diet decreases $\mathrm{BP}$ in hypertensive, obese and diabetic rats.

The differences in BP response might be explained by fibre dose, type of fibre consumed or better compliance with dietary supplements than with high-fibre diets ${ }^{(26)}$. Intake of fibre in the USA and many other Western countries is about $15 \mathrm{~g}$ daily $^{(27)}$, which is only half the amount recommended by the American Heart Association $\left(25-30 \mathrm{~g} / \mathrm{d}\right.$ from foods) ${ }^{(28)}$. It is estimated that Australian adults consume $18-25 \mathrm{~g}$ dietary fibre/ $\mathrm{d}^{(29)}$. This falls short of the Australian Government recommendations of $25 \mathrm{~g}$ dietary fibre/d for adult women and $30 \mathrm{~g}$ dietary fibre/d for men ${ }^{(30)}$. Given this large population segment with inadequate fibre intake, the demonstration of the beneficial effects of fibre on BP may have an impact on public health policy.

Overweight and obesity is associated with an increased risk of poor BP control ${ }^{(31)}$ and hyperlipidaemia. The augmentation index (AI) is an indicator of arterial stiffness and has been shown to be higher in those with hypercholesterolaemia ${ }^{(32)}$. It has been suggested that the increase in arterial stiffening may be associated with an increase in SBP and $\mathrm{DBP}^{(33-35)}$. Arterial stiffness is one of the major risk factors of $\mathrm{CVD}^{(36)}$, presenting an independent risk factor for this condition ${ }^{(36,37)}$. It is an age-related phenomenon ${ }^{(38,39)}$ and its progression is faster in patients with clusters of the metabolic syndrome ${ }^{(40-42)}$, resulting in luminal enlargement with wall thickening (remodelling) and a reduction in elastic properties (stiffening) at the level of large elastic arteries, namely arteriosclerosis $^{(43)}$. Besides the metabolic syndrome ${ }^{(44)}$, patients with hypertension $^{(45)}$ or diabetes ${ }^{(46)}$ exhibit increased carotid wall thickness and stiffness and this 'accelerated' arterial ageing is well confirmed to be a risk for $\mathrm{CVD}^{(47)}$. It is currently unknown whether fibre supplementation can improve arterial stiffness.

Some mechanisms have been suggested to explain the potential effect of dietary fibre intake on BP. Fibre seems to decrease the lipid profile by increasing intestinal viscosity ${ }^{(48)}$, therefore reducing bile acid absorption and promoting cholesterol catabolism ${ }^{(49)}$. A high fibre intake is associated with a decrease in total and LDL-cholesterol ${ }^{(50,51)}$, thereby potentially lowering the incidence of coronary disease ${ }^{(52)}$. It has been shown that serum cholesterol affects BP regulation ${ }^{(53)}$ by impairing endothelium-dependent dilation ${ }^{(54)}$. Interestingly, many hypertensive patients also appear to have increased serum cholesterol levels ${ }^{(55)}$. However, whether this is only a statistical association or it also implies a pathophysiological link is still under discussion ${ }^{(53)}$. Hypercholesterolaemia is associated with the loss of NO-induced vasodilation ${ }^{(56)}$ and the subsequent increase in $\mathrm{BP}^{(57)}$. There is evidence that cholesterol induces endothelial dysfunction even at normal or high-to-normal ranges by reducing the bioavailability of endothelium-derived $\mathrm{NO}^{(54,56)}$. Higher cholesterol levels may also be associated with more atherosclerotic vessels, not only in the carotid circulation by the increase in intimamedia thickness, but also in other arteries ${ }^{(53)}$. In addition, water-soluble fibre seems to reduce insulin resistance in both diabetic and healthy subjects ${ }^{(58,59)}$, which is suggested to be involved in the development of hypertension ${ }^{(60)}$. Hence, there is a possibility that fibre intake can also improve BP and endothelial function in overweight individuals.

Currently, there is a lack of studies on the effects of increasing dietary fibre intake on BP and arterial stiffness. A direct comparison between a group taking fibre supplementation alone and a group taking increased fibre by adopting a healthy eating pattern needs to be undertaken to reveal the effects from these different sources on chronic disease risk factors in overweight and obese individuals.

Psyllium husk fibre is a viscous, mostly water-soluble fibre and has been shown to be an effective supplement in adjunct to dietary intervention to control blood cholesterol, TAG and insulin levels in our previous studies ${ }^{(61,62)}$. Therefore, the aim of the present study was to investigate whether 12 weeks of additional dietary fibre intake in the form of a healthy diet or dietary fibre supplement (psyllium) would sustain improvements in vascular function and BP. 


\section{Methods}

\section{Subjects}

Overweight and obese individuals with a BMI between 25 and $40 \mathrm{~kg} / \mathrm{m}^{2}$ and aged between 18 and 65 years, presenting lipid profile and glucose/insulin levels in any range were recruited through local media (newspaper and television) from the community of Perth, Australia. Of ninety-four respondents, seventy-two were eligible to participate (thirty-seven men and thirty-five women) and commenced the study. Potential participants were screened by telephone and they attended at Curtin University to assess their suitability for the study, at which time the details of the study were explained. Exclusion criteria included smoking, lipid-lowering medication, use of steroids and other agents that may influence lipid metabolism, use of warfarin, diabetes mellitus, hypo- and hyperthyroidism, cardiovascular events within the last 6 months, psychological unsuitability, major systemic diseases, gastrointestinal problems, proteinuria, liver failure, renal failure, weight fluctuations over the past 6 months and vegetarianism. The present study was conducted according to the guidelines laid down in the Declaration of Helsinki and all procedures involving human subjects were approved by the Curtin University Human Research Ethics Committee (approval no. HREC 157/ 2004). All participants gave their written informed consent.

\section{Study design}

The present study was a randomised, single-blind, parallel design over a 12 -week period. The randomisation was done based on sex and alphabetical sequences of the surnames. The participants were allocated into two main groups of males and females and their lists were sorted based on their surnames alphabetically on a spreadsheet. The first name on each list was allocated into group 1, the second name into group 2 and the third name into group 3, and the same sequence of 1,2 and 3 was repeated for the rest of the names, respectively, to allocate the participants into three intervention groups randomly and to avoid any bias. The study participants were randomised to one of three groups: the control group ( $n$ 18), who consumed placebo with their usual diet; the fibre supplement group (FIB, $n$ 18), who consumed a fibre supplement with their usual diet; a healthy eating with placebo (HLT, $n$ 18), who consumed placebo with a healthy eating regimen. The fibre supplement consisted of psyllium serving, which is a soluble fibre gel-forming mucilage from the Plantago ovata plant whose bioactive fraction is a fibre composed of a highly branched arabinoxylan ${ }^{(63)}$. The participants were instructed to take $12 \mathrm{~g}$ of Metamucil (Metamucil; P\&G, Sydney, NSW, Australia) containing $7 \mathrm{~g}$ of psyllium, three times a day, in a total extra fibre ingestion of $21 \mathrm{~g}$ daily. A serving size of $12 \mathrm{~g}$ of Metamucil supplement (two rounded teaspoons = approximately $12 \mathrm{~g}$ ) contains approximately $7 \mathrm{~g}$ of dietary psyllium fibre, with the other remaining $5 \mathrm{~g}$ being made up of citric acid, aspartame, sunset yellow FCF CI 15985, maltodextrin, artificial and natural orange flavour and $\mathrm{Na}$. Psyllium has advantages over other types of soluble fibre because it is less readily fermented and therefore causes less flatulence and abdominal bloating ${ }^{(63)}$. It was used in the present study as it would be easily tolerated and is readily marketed worldwide. The placebo consisted of $12 \mathrm{~g}$ of breadcrumbs with flavouring, which provided $1.5 \mathrm{~g}$ of soluble fibre, presenting low energy and fibre content and similarity in texture and appearance to the psyllium supplement. The participants were asked to consume either the placebo or fibre supplement mixed with $250 \mathrm{ml}$ water, 5-10 min before breakfast, lunch and dinner.

The subjects attended a briefing session on how to complete food records and comply with the study protocol. Dietary intake over the course of the trial was monitored through the completion of $3 \mathrm{~d}$ weighed food diaries at baseline, 6 and 12 weeks. Ad libitum healthy eating plans, as designed by a registered dietitian, were prescribed for the HLT group for 12 weeks, which included healthy meals and snacks based on the Dietary Guidelines for Australian Adults ${ }^{(64)}$. These study participants were asked to record their food and drink intake for $3 \mathrm{~d}$ every 3 weeks to monitor the adherence to the guidelines ${ }^{(64)}$ and were given feedback and strategies if necessary. These participants were closely monitored to ensure their compliance with the dietary guidelines.

The participants in the control and FIB groups were asked to maintain their usual dietary intake for the duration of the study. To monitor compliance, all the participants were provided with a measuring cup to measure either the fibre supplement or the placebo, they were required to complete a diary to record their supplement consumption and they were asked to return the empty and non-empty containers of the supplements at their 6- and 12-week visits.

\section{Assessments}

Subjects were asked to visit the Curtin University for measurements, in a fasted state and wearing light clothing, on three occasions, one for baseline measures, at 6 weeks and at 12 weeks. Body weight (UM-018 Digital Scales; Tanita, Tokyo, Japan) was recorded in light clothing. Height was measured to the nearest $0 \cdot 1 \mathrm{~cm}$, without shoes, using a stadiometer (26SM $200 \mathrm{~cm}$; SECA, Hamburg, Germany). Waist circumference was measured in the standing position at the narrowest area between the lateral lower rib and the iliac crest. Hip measurement was taken at the largest circumference of the lower abdomen. The waist and hip measurements were taken three times at baseline, week 6 and week 12. The average at each time point was then reported. BP was measured with an automated, calibrated sphygmomanometer (Dinamap; Compact T, Critikon, Germany), with subjects in a supine position after resting for at least $10 \mathrm{~min}$. The measurements were taken on the same arm three times at $1 \mathrm{~min}$ intervals. These readings were then averaged.

\section{Pulse-wave analysis for augmentation index}

Pulse-wave analysis was used to assess arterial stiffness and vascular function using the SphygmoCor ${ }^{\mathrm{TM}}$ (AtCor Medical, Sydney, NSW, Australia). SphygmoCor is a non-invasive device that enables aortic root pressure to be measured 
during a normal clinical consultation. All pulse-wave analysis measurements were taken in a quiet, temperature-controlled room $\left(22^{\circ} \mathrm{C}\right)$, after a period of at least 5 min of rest. Patients fasted overnight and were asked to refrain from drinking drinks containing caffeine. Only high-quality readings, defined as an in-device quality index of $90 \%$ (derived from an algorithm including average pulse height, pulse height variation, diastolic variation and the maximum rate of rise of the peripheral waveform) and acceptable curves by visual inspection by the investigator, were included in the analysis.

Measurements were taken in the fasting state at baseline, week 6 and week 12. For each assessment, at least three measurements were taken and results were averaged. The AI is negative in healthy young people, but with ageing or increasing cardiovascular risk, arteries stiffen and the AI becomes increasingly positive. The AI was calculated using the following formula: AI $(\%)=$ augmentation pressure/ $(\mathrm{SBP}-\mathrm{DBP}) \times 100$. The reference range provided by the Sphygmocor software (AtCor Medical) was determined from an analysis of 405 healthy individuals in a study by Wilkinson et al. ${ }^{(65)}$. Mean population AI at age 50 years was $18.43(90 \%$ CI $-0 \cdot 17,39 \cdot 97) \%$. All measurements were performed by the same operator. These measurements were taken three times with a quality index of at least $90 \%$ or over and the readings were averaged.

\section{Statistical analysis}

Statistical analysis was undertaken using SPSS 11 for Windows (SPSS, Inc., Chicago, IL, USA). Data are expressed as means with their standard errors and assessed for normality to ensure that the assumptions of the analysis are met. Data were analysed using multivariate ANOVA with baseline values considered as covariates. If significant between-group effects were present, post hoc comparisons between the treatment groups were made using the least significant difference method. Statistical significance was considered at $P<0 \cdot 05$.

A sample size of at least fifteen subjects per group will provide sufficient power $(0.90 \%)$ to detect an estimated within- and between-group effect size of 0.56 at a $5 \%$ significance level. Recruiting a total of seventy-two subjects ( $n$ 18) will allow for $20 \%$ dropouts.

The present clinical trial was registered with the Australian New Zealand Clinical Trials Registry. The registration no. is ACTRN12609000540213 and trial web address is https:// www.anzctr.org.au/ACTRN12609000540213.aspx

\section{Results}

\section{Subjects}

A total of seventy-two individuals were randomly assigned to one of the three test groups: control, FIB or HLT. Of these, fifteen participants withdrew from the study due to unrelated illness, work commitments, poor compliance and personal reasons. The remaining fifty-seven participants (twenty-five males and thirty-two females) completed the 12-week study (control: $n$ 15; FIB: $n$ 16; HLT: $n$ 12). Baseline values of clinical and vascular characteristics in the three treatment arms were not significantly different (Table 1). Although there were some reports of minor bloating initially, all subjects tolerated the supplements well with no adverse effects reported.

\section{Diet analysis}

The self-reported food and drink intake at baseline, 6 and 12 weeks is shown in Table 2 . There were no significant differences in total energy, carbohydrate, protein, total fat, saturated fat, monounsaturated fat, polyunsaturated fat and fibre between the three groups at baseline.

Fibre intake was higher $(P<0 \cdot 001)$ in the FIB group by $20.5 \mathrm{~g}$ and in the HLT group by $11 \mathrm{~g}$ at week 12 when compared with the control group. Fibre intake in the FIB group was also higher $(P<0 \cdot 001)$ than the HLT group by $9 \cdot 6 \mathrm{~g}$ at week 12 .

Total fat intake was lower $(P<0.001)$ in the HLT group by $6 \%$ at week 6 and by $24 \%$ at week 12 when compared with the control group. When compared with the FIB group, total fat intake was lower $(P<0.001$ and $P=0.020$, respectively) in the HLT group by $10 \%$ at week 6 and lower $(P<0.001)$ by $18 \%$ at week 12. Saturated fat intake was increased $(P<0.05)$ in the control group at 12 weeks when compared with baseline. The HLT group also showed an increase $(P<0.05)$ in saturated fat at 12 weeks when compared with baseline. Saturated fat intake was reduced $(P=0.029)$ by $26 \%$ in the HLT group when compared with the control group at week 12 . Polyunsaturated fat intake was also lower $(P=0.018)$ in the HLT group by $23 \%$ when compared with the control group at week 12. Monounsaturated fat intake in the HLT group was lower $(P=0.008)$ by $24 \%$ at week 6 and lower $(P=0 \cdot 047)$ by $20 \%$ at week 12 when compared with the control group.

\section{Effects of fibre on blood pressure}

Resting BP was measured in the fasting state, at baseline, week 6 and week 12, and there were no significant differences in SBP or DBP. Between-group analysis revealed that SBP was

Table 1. Clinical and vascular characteristics of subjects at baseline (Mean values with their standard errors) ${ }^{*}$

\begin{tabular}{|c|c|c|c|c|c|c|}
\hline \multirow{2}{*}{$\begin{array}{l}\text { Groups... } \\
\text { Characteristics }\end{array}$} & \multicolumn{2}{|c|}{ Control } & \multicolumn{2}{|c|}{ FIB } & \multicolumn{2}{|c|}{ HLT } \\
\hline & Mean & SEM & Mean & SEM & Mean & SEM \\
\hline Age (years) & $44 \cdot 8$ & 1.6 & 41.3 & $2 \cdot 3$ & $40 \cdot 8$ & 3.0 \\
\hline Weight (kg) & $97 \cdot 8$ & $3 \cdot 2$ & 101.6 & 3.0 & 101.9 & 4.6 \\
\hline BMI $\left(\mathrm{kg} / \mathrm{m}^{2}\right)$ & 33.7 & 1.0 & 34.0 & 0.9 & 36.1 & 1.5 \\
\hline$W C(\mathrm{~cm})$ & $104 \cdot 8$ & 3.0 & $110 \cdot 2$ & 3.5 & 110.9 & 3.5 \\
\hline Waist:hip ratio & 0.9 & $2 \cdot 1$ & 0.9 & $2 \cdot 8$ & 0.8 & $2 \cdot 7$ \\
\hline SBP (mmHg) & $114 \cdot 3$ & $2 \cdot 7$ & $115 \cdot 0$ & 2.5 & 111.3 & 3.0 \\
\hline $\mathrm{DBP}(\mathrm{mmHg})$ & $66 \cdot 3$ & $2 \cdot 3$ & $66 \cdot 4$ & $2 \cdot 6$ & 62.6 & $2 \cdot 6$ \\
\hline $\mathrm{Al}(\%)$ & $40 \cdot 0$ & $7 \cdot 0$ & $26 \cdot 6$ & $4 \cdot 3$ & $30 \cdot 1$ & $5 \cdot 2$ \\
\hline
\end{tabular}

FIB, fibre supplement group; HLT, healthy eating with placebo; WC, waist circumference; SBP, systolic blood pressure; DBP, diastolic blood pressure; AI, augmentation index.

* Mean values were not significantly different between subject characteristics at screening. 
Table 2. Reported dietary intake data assessed by weighed food records $†$

(Mean values with their standard errors, $n 57$ )

\begin{tabular}{|c|c|c|c|c|c|c|}
\hline & \multicolumn{2}{|c|}{ Baseline } & \multicolumn{2}{|c|}{ Week 6} & \multicolumn{2}{|c|}{ Week 12} \\
\hline & Mean & SEM & Mean & SEM & Mean & SEM \\
\hline \multicolumn{7}{|l|}{ Total EI } \\
\hline Control & $8054 \cdot 1$ & $428 \cdot 4$ & 8325 & $629 \cdot 2$ & $8165 \cdot 7^{a}$ & $509 \cdot 2$ \\
\hline FIB & 8375.9 & $467 \cdot 1$ & $7817 \cdot 4$ & 359.6 & $7756 \cdot 8^{a, b}$ & $369 \cdot 2$ \\
\hline HLT & $8266 \cdot 3$ & 479.2 & $7807 \cdot 6$ & $422 \cdot 7$ & $7255 \cdot 4^{\mathrm{a}, \mathrm{b}}$ & $325 \cdot 4$ \\
\hline \multicolumn{7}{|c|}{ Carbohydrate (\%EI) } \\
\hline Control & 43.8 & $2 \cdot 4$ & 43.8 & $2 \cdot 4$ & 44.5 & $2 \cdot 2$ \\
\hline FIB & $45 \cdot 9$ & $2 \cdot 0$ & 45.5 & 1.5 & $46 \cdot 3$ & $2 \cdot 1$ \\
\hline HLT & $45 \cdot 8$ & 1.7 & $47 \cdot 1$ & 1.7 & 49.9 & $2 \cdot 1$ \\
\hline \multicolumn{7}{|c|}{ Protein intake (\%El) } \\
\hline Control & $18 \cdot 3$ & 1.3 & $18 \cdot 2$ & 1.2 & $18 \cdot 2$ & 1.0 \\
\hline FIB & $17 \cdot 7$ & $1 \cdot 1$ & 18.5 & 1.0 & $19 \cdot 3$ & $1 \cdot 1$ \\
\hline HLT & 17.4 & $1 \cdot 1$ & $19 \cdot 3$ & 1.1 & $21.8^{*}$ & 1.2 \\
\hline \multicolumn{7}{|c|}{ Fat intake (\%El) } \\
\hline Control & $37 \cdot 8$ & 1.9 & $37.8^{\mathrm{a}}$ & $2 \cdot 1$ & $37.1^{\mathrm{a}}$ & $2 \cdot 1$ \\
\hline FIB & $36 \cdot 2$ & 1.5 & $35.9^{\mathrm{a}}$ & 0.9 & $34.3^{\mathrm{a}}$ & 1.7 \\
\hline HLT & 36.6 & 1.6 & $32 \cdot 3^{\star b}$ & 1.4 & $28 \cdot 2^{* b}$ & $1 \cdot 1$ \\
\hline \multicolumn{7}{|c|}{ SFA intake (\%El) } \\
\hline Control & 14.4 & $1 \cdot 2$ & $17 \cdot 8$ & 1.3 & $19 \cdot 7^{\star a}$ & 1.9 \\
\hline FIB & 14.6 & 1.0 & $17 \cdot 0$ & 0.8 & $17 \cdot 4^{\mathrm{a}, \mathrm{b}}$ & $1 \cdot 1$ \\
\hline HLT & $12 \cdot 4$ & 0.8 & $16 \cdot 1^{*}$ & 1.0 & $14 \cdot 6^{\star b}$ & $1 \cdot 1$ \\
\hline \multicolumn{7}{|c|}{ MUFA intake (\%EI) } \\
\hline Control & $14 \cdot 2$ & 0.8 & $13 \cdot 7^{\mathrm{a}}$ & $1 \cdot 1$ & $11.4^{\star a}$ & 1.0 \\
\hline FIB & $14 \cdot 2$ & 0.7 & $13 \cdot 0^{\mathrm{a}}$ & 0.8 & $11 \cdot 3^{\star a, b}$ & 0.7 \\
\hline HLT & 14.5 & 0.6 & $10 \cdot 4^{\star b}$ & 1.0 & $9 \cdot 0^{* \mathrm{~b}}$ & 0.6 \\
\hline \multicolumn{7}{|c|}{ PUFA intake (\%EI) } \\
\hline Control & 9.2 & 1.2 & $6 \cdot 3^{*}$ & 0.6 & $6 \cdot 0^{\star a}$ & 0.5 \\
\hline FIB & 7.5 & 0.7 & $5 \cdot 9^{*}$ & 0.3 & $5 \cdot 7^{\star a, b}$ & 0.5 \\
\hline HLT & $9 \cdot 8$ & 1.3 & $5 \cdot 9^{*}$ & 0.6 & $4 \cdot 6^{\star \mathrm{b}}$ & 0.4 \\
\hline \multicolumn{7}{|c|}{ Fibre intake $(\mathrm{g})$} \\
\hline Control & 23.7 & $1 \cdot 2$ & $19 \cdot 7^{\star \mathrm{a}}$ & 0.6 & $19 \cdot 7^{\star a}$ & 0.6 \\
\hline FIB & 23.9 & 1.0 & $38 \cdot 1^{\star b}$ & $2 \cdot 0$ & $40 \cdot 3^{\star c}$ & 1.9 \\
\hline HLT & $24 \cdot 0$ & 1.0 & $31.6^{\mathrm{c}}$ & $1 \cdot 2$ & $30 \cdot 7^{b}$ & 0.6 \\
\hline
\end{tabular}

El, energy intake; FIB, fibre supplement group; HLT, healthy eating with placebo.

${ }_{\mathrm{a}, \mathrm{b}, \mathrm{c}}$ Mean values with unlike superscript letters were significantly different between the groups at 6 and 12 weeks $(P<0.05)$.

* Mean values were significantly different from baseline $(P<0.05)$.

$\dagger$ Nutritional data recorded in $3 \mathrm{~d}$ food diaries at baseline, week 6 and week 12

lower in the FIB group by $7 \%(P=0.04)$ compared with the control group at week 6. SBP was lower in the HLT group by $9 \%(P=0.02)$ compared with the control group at week 12 (Fig. 1(a)). There were no differences in SBP between the other intervention groups at week 12 compared with the control group.

Analysis between the groups at week 6 showed that DBP was lower by $7 \%(P=0.04)$ and $3 \%(P=0.04)$ in the FIB and HLT groups, respectively, compared with the control group. When DBP results were analysed at week 12 , there was no difference between the groups (Fig. 1(b)).

\section{Effects of fibre on vascular function}

Central AI was measured in the fasting state, at baseline, week 6 and week 12, and there were no significant differences within the groups at 6 or 12 weeks from baseline. Analysis of data between the groups showed that the AI was lower by $22 \%$ in the FIB group compared with the control group $(P=0.02)$ at week 6 , but it was not different between the groups at week 12 (Fig. 2).

\section{Discussion}

The aim of the present study was to investigate whether 12 weeks of consumption of a fibre supplement or a healthy eating diet would induce improvements to BP and arterial stiffness in overweight and obese individuals compared with a standard diet. SBP was significantly lower in the FIB group at week 6 compared with the control group. However, these changes were temporary and the only significant difference in SBP at week 12 was between the HLT and control groups. DBP was lower in the FIB group compared with the control group at week 6 , but this did not last and there were no significant differences in DBP between the groups at the end of the study. Collectively, the present study showed that fibre supplementation in the form of psyllium had no effect on BP and endothelial function; however, increased fibre intake in the form of a healthy diet was beneficial to lowering BP.

Some mechanisms have been suggested to explain the potential effect of dietary fibre intake on BP, since dietary fibre presents numerous effects on the digestion and absorption of foods. The mechanism by which fibre lowers CVD 

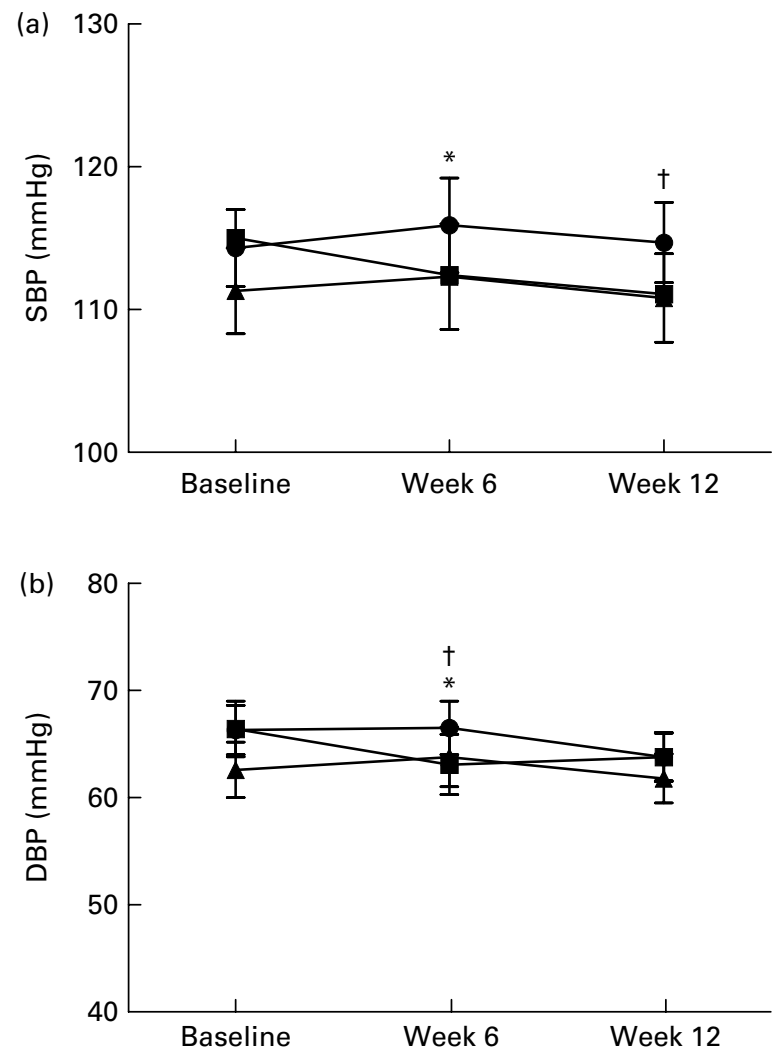

Fig. 1. Blood pressure levels at baseline, week 6 and week 12 between the three intervention groups (control (--) $n 15$, fibre supplement group (FIB; $-\mathbf{\square}-) n 16$, healthy eating with placebo (HLT; $-\mathbf{\Delta}-) n 12)$. (a) Systolic blood pressure (SBP, $\mathrm{mmHg}$ ) and (b) diastolic blood pressure (DBP, $\mathrm{mmHg}$ ). Values are means, with their standard errors represented by vertical bars. ${ }^{*}$ Mean values were significantly different between the FIB $v$. control groups $(P<0.05)$. †Mean values were significantly different between the HLT $v$. control groups $(P<0.05)$.

risk remains to be determined; however, studies have suggested that fibre decreases the lipid profile by increasing intestinal viscosity ${ }^{(48)}$, therefore reducing bile acid absorption and promoting cholesterol catabolism ${ }^{(49)}$. In particular, a high fibre intake is associated with a decrease in the serum concentrations of total and LDL-cholesterol ${ }^{(50,51)}$ and, at the same time, with a lower incidence of coronary disease ${ }^{(52)}$. In addition, water-soluble fibre seems to reduce insulin resistance and insulin plasma concentrations in both diabetic and healthy subjects ${ }^{(58,59)}$. Insulin resistance is implicated in the pathogenic mechanism for the development of hypertension ${ }^{(60)}$.

Although little is known about the potential mechanisms by which dietary fibre may lower BP, there are a few studies investigating the beneficial effects of dietary fibre intake on BP. Studies with animals have suggested that a high-fibre diet decreases BP in hypertensive, obese and diabetic rats ${ }^{(23,24)}$. In fact, prospective observational studies that have analysed the relationship between dietary fibre intake and the risk of developing hypertension have indicated that an increased consumption of dietary fibre intake is inversely related to BP and may contribute to prevent hypertension ${ }^{(17,18,31,66)}$, in both hypertensive or normotensive subjects.

In the present study, SBP and DBP were lower in the FIB group compared with the control group at week 6. However, this change appears to have been temporary as there were no significant differences in SBP and DBP between any groups at week 12. This discrepancy may have been due to lower $\mathrm{BP}$ in the control group at week 12. This may be due to day-to-day variability in $\mathrm{BP}^{(67)}$ or to some other factors such as psychological stress and mood which can affect BP levels ${ }^{(68)}$. However, the reason why this would occur in the control group and not affect the other groups in the present study is unknown. The other possibility for no difference in BP between the groups after 12 weeks of study was that the average $\mathrm{BP}$ of the groups was within the healthy range and it is hard to highlight the BP differences in normotensive participants, as dietary fibre usually makes small changes in BP in these groups ${ }^{(66,69)}$.

A good body of evidence supports the adoption of a diet high in fruits, vegetables and fibre and low in fat, which suggests that such a diet helps protect against CVD and diabetes $^{(70)}$. Thus, the psyllium supplement consumed in the present study may not be as effective as the multiple sources of fibre from consuming non-viscous fibres and pectin from whole grain, fruit and vegetables. Furthermore, there is evidence that soluble fibre improves mineral absorption in the digestive system ${ }^{(71,72)}$, which may have a favourable effect on BP. In the Dietary Approaches to Stop Hypertension trial, the effect of dietary patterns on BP was assessed. The fruitsand-vegetables diet provided $\mathrm{K}, \mathrm{Mg}$ and high amounts of fibre, and resulted in $\mathrm{BP}$ reductions of $2 \cdot 8 / 1 \cdot 1 \mathrm{mmHg}$ compared with a typical US diet. Other studies have provided support for an increased effect of soluble fibre on $\mathrm{BP}^{(14)}$.

In addition, several other mechanisms for a potential effect of dietary fibre intake on BP have been hypothesised. Dietary fibre has numerous effects on the digestion and absorption of foods and can change the glycaemic index of foods, thereby altering insulin response. Insulin plays a role in BP regulation and dietary fibre can modify the insulin and vascular endothelial function ${ }^{(73)}$. Insulin resistance and its concomitant compensatory hyperinsulinaemia have been suggested as major underlying pathogenetic mechanisms for the development of hypertension ${ }^{(74)}$. In this way, the effectiveness of both

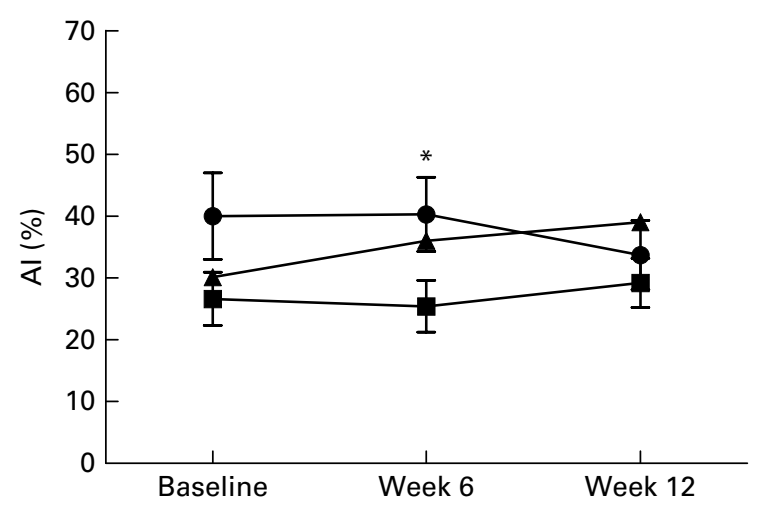

Fig. 2. Central augmentation index (Al; \%) at baseline, week 6 and week 12 between the three intervention groups (control (- - $n$ 15, fibre supplement group (FIB; $-\mathbf{-}-) n 16$, healthy eating with placebo $(-\boldsymbol{\Delta}-) n 12)$. Values are means, with their standard errors represented by vertical bars. * Mean values were significantly different between the FIB $v$. control groups $(P<0.05)$. 
soluble and insoluble fibres in reducing insulin resistance and insulin levels in both diabetic and healthy persons ${ }^{(75,76)}$ would contribute to treating or preventing hypertension. Our previous studies showed that simply adding psyllium fibre supplementation to a normal diet was sufficient to see improvements in total and LDL-cholesterol as well as body weight, BMI and percentage of body fat compared with the control group. However, there was no effect of psyllium fibre supplementation on insulin levels, but fasting insulin levels decreased at week 12 in the HLT group by $24 \%(P=0.03)$ compared with baseline ${ }^{(61)}$. When compared with the control group at week 12, insulin levels decreased in the HLT group by $21 \%(P=0 \cdot 001)$. Given that insulin plays a regulatory role on $\mathrm{BP}$, the lack of change in insulin levels in the FIB group may partially explain why fibre supplementation did not affect BP in the FIB group.

In subjects with the metabolic syndrome, the $\mathrm{AI}$, an indicator of arterial stiffness, was shown to be significantly higher than in subjects with normal weight for the same age and mean arterial pressure ${ }^{(77,78)}$. Thus, this measurement is one of the most appropriate for repeat determinations in clinical studies. Studies have shown an inverse relationship between healthy eating and arterial stiffness ${ }^{(79)}$. Compared with a low-fibre meal (12-16\% carbohydrate containing $0.5 \mathrm{~g}$ cereal fibre), a mix of high-carbohydrate, high-fibre meal (69-76\% carbohydrate containing $19 \mathrm{~g}$ cereal fibre) has been shown to improve flow-mediated dilation through increasing NO release in subjects with the metabolic syndrome ${ }^{(80)}$. However, the role of dietary fibre in preventing and improving arterial stiffness in the short and long term has yet to be fully elucidated. Endothelial function has an important role in regulating BP and vascular resistance ${ }^{(81)}$. Consumption of psyllium husk has been shown to alter the endothelial function and SBP in Zucker rats ${ }^{(23)}$. The hypotensive effect of psyllium was attributed, at least in part, to its ability to inhibit intestinal absorption of $\mathrm{Na}$ and improve the endothelial function by improving hyperlipidaemia and decreasing the inflammatory markers ${ }^{(24)}$. The present data analysis showed that the central AI did not change over 12 weeks of intervention from the baseline in any group. Data analysis between the groups showed that the AI was lower by $22 \%$ in the FIB group compared with the control group at week 6; however, this did not last and there were no significant differences in the AI between the groups at the end of the study. This suggests that the mechanism of reduction of BP following dietary supplementation with psyllium is related to specific alterations in endothelial function. The present study is the first to investigate the effect of psyllium husk on arterial stiffness in human subjects. There is some evidence from animal studies which supports the beneficial effects of psyllium on endothelial function, such as diabetic rats fed daily with psyllium husk that showed improvement on endothelial function and, consequently, on SBP by altering the endothelium response to acetylcholine and improving inflammatory markers ${ }^{(23)}$. Markers of the endothelial function such as NO were not measured in the present study, but it may be one of the mechanisms that altered BP temporarily in the intervention groups.
The amount of fibre supplementation of $21 \mathrm{~g}$ psyllium/d, as well as the duration of the study of 12 weeks, was considered suitable to detect differences in BP. Our choices were based on previous studies confirming this information. A metaanalysis based on the evidence from twenty-five randomised, controlled clinical trial, conducted in 1477 participants with or without hypertension observed that a fibre intake of $7 \cdot 2-18.9 \mathrm{~g} / \mathrm{d}$ reduced significantly both $\mathrm{SBP}(-2.3 \mathrm{mmHg})$ and DBP $(-1.97 \mathrm{mmHg})$, respectively. Trials whose duration was 8 weeks or more in length showed a significant decrease in SBP and DBP $(-3.12$ and $-1.14 \mathrm{mmHg}$, respectively) mainly in hypertensive subjects but also in normotensive subjects $^{(18)}$. Therefore, the dose of fibre and the duration of the study length used in the present study are well above the range used in previous trials showing the beneficial effects of fibre on BP.

The present study has some limitations. Hypertensive subjects on various medications such as lipid lowering, hypertension, etc. had responded to our newspaper article but were then excluded given the confounding effects of these medications. Therefore, the average BP of the participants in the present study was within the healthy range. This may be the reason why no differences in BP was observed between the groups after 12 weeks of study, as it is hard to highlight the BP differences in normotensive participants. Dietary fibre usually makes only small changes in $\mathrm{BP}$ in normotensive groups $^{(66,69)}$.

The lack of significant differences in SBP and DBP between the FIB and HLT groups at the end of the study may also be due to lower BP in the control group at week 12, due to day-to-day variability in $\mathrm{BP}^{(67)}$ or due to some other factors such as psychological stress and mood which can affect BP levels ${ }^{(68)}$. However, the reason why this would occur in the control group and not affect the other groups in the present study is unknown. Based on the variability in BP observed in the present study, the trial had very low power to detect clinically meaningful changes.

The lack of any improvement in arterial stiffness in the FIB and HLT groups may be due to the normal BP of the individuals and the short duration of the study. A meta-analysis of studies about the effects of healthy eating and dietary fibre on $\mathrm{BP}$ and endothelial function showed that these effects are more prominent in hypertensive individuals compared with normotensive individuals, and significant changes are usually measurable after a few months of intervention ${ }^{(18)}$. Future studies with hypertensive subjects would clarify whether these results persist or not.

In summary, the present study did not show any improvements in BP or vascular function in overweight and obese individuals with psyllium fibre supplementation, although a healthy diet decreased SBP. There may be factors in a healthy diet affecting BP, which may not be related to an increase in fibre. Further studies are required in hypertensive overweight and obese patients to examine whether psyllium fibre supplementation can have beneficial effects on vascular health and BP. 


\section{Acknowledgements}

The present trial was partially funded by the ATN Centre for Metabolic Fitness. A. K. coordinated the trial, conducted data collection, statistical analysis and provided input into the manuscript. C. B. provided input into the manuscript. S. D. supervised the statistical analysis. S. R.-B. contributed to the writing of the manuscript. S. P. conceived and designed the study, supervised the study and the statistical analysis and mentored the script. The authors declare that they have no conflicts of interest.

\section{References}

1. WHO (2002) Nutrition: Controlling the Global Obesity Epidemic. Geneva: World Health Organization.

2. Popkin BM (2001) The nutrition transition and obesity in the developing world. J Nutr 131, 871S-873S.

3. Isomaa B, Almgren P, Tuomi T, et al. (2001) Cardiovascular morbidity and mortality associated with the metabolic syndrome. Diabetes Care 24, 683-689.

4. Grundy SM (2004) Obesity, metabolic syndrome, and cardiovascular disease. J Clin Endocrinol Metab 89, 2595-2600.

5. Cook S, Hugli O, Egli M, et al. (2003) Clustering of cardiovascular risk factors mimicking the human metabolic syndrome $\mathrm{X}$ in eNOS null mice. Swiss Med Wkly 133, 360-363.

6. Appel LJ, Brands MW, Daniels SR, et al. (2006) Dietary approaches to prevent and treat hypertension: a scientific statement from the American Heart Association. Hypertension 47, 296-308.

7. Streppel MT, Arends LR, van't Veer P, et al. (2005) Dietary fiber and blood pressure: a meta-analysis of randomized placebo-controlled trials. Arch Intern Med 165, 150-156.

8. Greger JL (1999) Nondigestible carbohydrates and mineral bioavailability. J Nutr 129, 1434S-1435S.

9. Coudray C, Demigne C \& Rayssiguier Y (2003) Effects of dietary fibers on magnesium absorption in animals and humans. J Nutr 133, 1-4.

10. He J, Klag MJ, Whelton PK, et al. (1995) Dietary macronutrients and blood pressure in southwestern China. J Hypertens 13, 1267-1274.

11. Stamler J, Caggiula AW \& Grandits GA (1997) Relation of body mass and alcohol, nutrient, fiber, and caffeine intakes to blood pressure in the special intervention and usual care groups in the Multiple Risk Factor Intervention Trial. Am J Clin Nutr 65, 338S-365s.

12. Birketvedt GS, Aaseth J, Florholmen JR, et al. (2000) Longterm effect of fibre supplement and reduced energy intake on body weight and blood lipids in overweight subjects. Acta Medica (Hradec Kralove) 43, 129-132.

13. Eliasson K, Ryttig KR, Hylander B, et al. (1992) A dietary fibre supplement in the treatment of mild hypertension. A randomized, double-blind, placebo-controlled trial. J Hypertens 10, 195-199.

14. Streppel MT, Arends LR, van 't Veer P, et al. (2005) Dietary fiber and blood pressure: a meta-analysis of randomized placebo-controlled trials. Arch Intern Med 165, 150-156.

15. Ascherio A, Rimm EB, Giovannucci EL, et al. (1992) A prospective study of nutritional factors and hypertension among US men. Circulation 86, 1475-1484.

16. WHO (2003) Diet, Nutrition and the Prevention of Chronic Diseases. Geneva: World Health Organization and FAO.

17. Burke V, Hodgson JM, Beilin LJ, et al. (2001) Dietary protein and soluble fiber reduce ambulatory blood pressure in treated hypertensives. Hypertension 38, 821-826.
18. Whelton SP, Hyre AD, Pedersen B, et al. (2005) Effect of dietary fiber intake on blood pressure: a meta-analysis of randomized, controlled clinical trials. J Hypertens $\mathbf{2 3}$, 475-481.

19. Solum TT, Ryttig KR, Solum E, et al. (1987) The influence of a high-fibre diet on body weight, serum lipids and blood pressure in slightly overweight persons. A randomized, double-blind, placebo-controlled investigation with diet and fibre tablets (DumoVital). Int J Obes 11, Suppl. 1, 67-71.

20. Schlamowitz P, Halberg T, Warnoe O, et al. (1987) Treatment of mild to moderate hypertension with dietary fibre. Lancet 2, 622-623.

21. Singh RB, Rastogi SS, Singh NK, et al. (1993) Can guava fruit intake decrease blood pressure and blood lipids? J Hum Hypertens 7, 33-38.

22. He J, Streiffer RH, Muntner P, et al. (2004) Effect of dietary fiber intake on blood pressure: a randomized, doubleblind, placebo-controlled trial. J Hypertens 22, 73-80.

23. Galisteo M, Sanchez M, Vera R, et al. (2005) A diet supplemented with husks of Plantago ovata reduces the development of endothelial dysfunction, hypertension, and obesity by affecting adiponectin and TNF-\{alpha\} in obese Zucker rats. J Nutr 135, 2399-2404.

24. Obata K, Ikeda K, Yamasaki M, et al. (1998) Dietary fiber, psyllium, attenuates salt-accelerated hypertension in strokeprone spontaneously hypertensive rats. $J$ Hypertens $\mathbf{1 6}$, 1959-1964.

25. Li J, Wang J, Kaneko T, et al. (2004) Effects of fiber intake on the blood pressure, lipids, and heart rate in Goto Kakizaki rats. Nutrition 20, 1003-1007.

26. He J \& Whelton PK (1999) Effect of dietary fiber and protein intake on blood pressure: a review of epidemiologic evidence. Clin Exp Hypertens 21, 785-796.

27. Alaimo K, McDowell MA, Briefel RR, et al. (1994) Dietary intake of vitamins, minerals, and fiber of persons ages 2 months and over in the United States: Third National Health and Nutrition Examination Survey, Phase 1, 1988-91. Adv Data 1-28.

28. Marlett JA, McBurney MI \& Slavin JL (2002) Position of the American Dietetic Association: health implications of dietary fiber. J Am Diet Assoc 102, 993-1000.

29. State of Victoria (2008) Fibre in Food. Victoria, Australia: Department of Primary Industries.

30. National Health and Medical Research Council (2005) Nutrient reference values for Australia and New Zealand [NHaMR Council, editor]. Canberra: National Health and Medical Research Council.

31. Hense HW (2004) Once at risk-forever at risk? The long-term impact of cardiovascular risk factors on death. Eur J Epidemiol 19, 409-410.

32. Wilkinson IB, Prasad K, Hall IR, et al. (2002) Increased central pulse pressure and augmentation index in subjects with hypercholesterolemia. J Am Coll Cardiol 39, 1005-1011.

33. Blacher J \& Safar ME (2005) Large-artery stiffness, hypertension and cardiovascular risk in older patients. Nat Clin Pract Cardiovasc Med 2, 450-455.

34. Safar ME (2001) Systolic blood pressure, pulse pressure and arterial stiffness as cardiovascular risk factors. Curr Opin Nephrol Hypertens 10, 257-261.

35. Tomiyama H, Hashimoto H, Hirayama Y, et al. (2006) Synergistic acceleration of arterial stiffening in the presence of raised blood pressure and raised plasma glucose. Hypertension 47, 180-188. 
36. Qureshi G, Brown R, Salciccioli L, et al. (2007) Relationship between aortic atherosclerosis and non-invasive measures of arterial stiffness. Atherosclerosis 195, e190-e194.

37. Casey DP, Pierce GL, Howe KS, et al. (2007) Effect of resistance training on arterial wave reflection and brachial artery reactivity in normotensive postmenopausal women. Eur $J$ Appl Physiol 100, 403-408.

38. Greenwald SE (2007) Ageing of the conduit arteries. J Pathol 211, $157-172$.

39. McEniery CM, Wilkinson IB \& Avolio AP (2007) Age, hypertension and arterial function. Clin Exp Pharmacol Physiol 34, 665-671.

40. Ostmark E, Harrisson S, Wooley KL, et al. (2007) Comb polymers prepared by ATRP from hydroxypropyl cellulose. Biomacromolecules 8, 1138-1148.

41. McEniery CM \& Cockcroft JR (2007) Does arterial stiffness predict atherosclerotic coronary events? Adv Cardiol 44, $160-172$.

42. Safar ME, Thomas F, Blacher J, et al. (2006) Metabolic syndrome and age-related progression of aortic stiffness. J Am Coll Cardiol 47, 72-75.

43. Izzo JL Jr \& Shykoff BE (2001) Arterial stiffness: clinical relevance, measurement, and treatment. Rev Cardiovasc Med 2, 29-34, 37-40.

44. Scuteri A, Najjar SS, Muller DC, et al. (2004) Metabolic syndrome amplifies the age-associated increases in vascular thickness and stiffness. J Am Coll Cardiol 43, 1388-1395.

45. Arnett DK, Tyroler HA, Burke G, et al. (1996) Hypertension and subclinical carotid artery atherosclerosis in blacks and whites. The Atherosclerosis Risk in Communities Study. ARIC Investigators. Arch Intern Med 156, 1983-1989.

46. Amar J, Ruidavets JB, Chamontin B, et al. (2001) Arterial stiffness and cardiovascular risk factors in a populationbased study. J Hypertens 19, 381-387.

47. Benetos A, Waeber B, Izzo J, et al. (2002) Influence of age, risk factors, and cardiovascular and renal disease on arterial stiffness: clinical applications. Am J Hypertens 15, 1101-1108.

48. Jenkins DJ, Kendall CW, Axelsen M, et al. (2000) Viscous and nonviscous fibres, nonabsorbable and low glycaemic index carbohydrates, blood lipids and coronary heart disease. Curr Opin Lipidol 11, 49-56.

49. Salas-Salvado J, Bullo M, Perez-Heras A, et al. (2006) Dietary fibre, nuts and cardiovascular diseases. BrJ Nutr 96, Suppl. 2, S46-S51.

50. Jenkins DJ, Wolever TM, Rao AV, et al. (1993) Effect on blood lipids of very high intakes of fiber in diets low in saturated fat and cholesterol. $N$ Engl J Med 329, 21-26.

51. Brown L, Rosner B, Willett WW, et al. (1999) Cholesterollowering effects of dietary fiber: a meta-analysis. Am J Clin Nutr 69, 30-42.

52. Bingham SA, Day NE, Luben R, et al. (2003) Dietary fibre in food and protection against colorectal cancer in the European Prospective Investigation into Cancer and Nutrition (EPIC): an observational study. Lancet 361, 1496-1501.

53. Ferrara LA, Guida L, Iannuzzi R, et al. (2002) Serum cholesterol affects blood pressure regulation. J Hum Hypertens $\mathbf{1 6}$, $337-343$.

54. Creager MA, Cooke JP, Mendelsohn ME, et al. (1990) Impaired vasodilation of forearm resistance vessels in hypercholesterolemic humans. J Clin Invest 86, 228-234.

55. Laurenzi M, Mancini M, Menotti A, et al. (1990) Multiple risk factors in hypertension: results from the Gubbio study. J Hypertens Suppl 8, S7-S12.
56. Hayakawa H \& Raij L (1999) Relationship between hypercholesterolaemia, endothelial dysfunction and hypertension. J Hypertens 17, 611-619.

57. Vallance P, Collier J \& Moncada S (1989) Effects of endothelium-derived nitric oxide on peripheral arteriolar tone in man. Lancet ii, 997-1000.

58. Anderson JW, Zeigler JA, Deakins DA, et al. (1991) Metabolic effects of high-carbohydrate, high-fiber diets for insulin-dependent diabetic individuals. Am J Clin Nutr 54, 936-943.

59. Fukagawa NK, Anderson JW, Hageman G, et al. (1990) Highcarbohydrate, high-fiber diets increase peripheral insulin sensitivity in healthy young and old adults. Am J Clin Nutr 52, 524-528.

60. Ferrannini E, Buzzigoli G, Bonadonna R, et al. (1987) Insulin resistance in essential hypertension. $N$ Engl J Med 317, 350-357.

61. Pal S, Khossousi A, Binns C, et al. (2011) The effect of a fibre supplement compared to a healthy diet on body composition, lipids, glucose, insulin and other metabolic syndrome risk factors in overweight and obese individuals. Br J Nutr 105, 90-100.

62. Khossousi A, Binns CW, Dhaliwal Ss, et al. (2008) The acute effects of psyllium on postprandial lipaemia and thermogenesis in overweight and obese men. Br J Nutr 99, 1068-1075.

63. Blackwood AD, Salter J, Dettmar PW, et al. (2000) Dietary fibre, physicochemical properties and their relationship to health. $J$ R Soc Promot Health 120, 242-247.

64. Lee YM, Skurk T, Hennig M, et al. (2007) Effect of a milk drink supplemented with whey peptides on blood pressure in patients with mild hypertension. Eur J Nutr 46, 21-27.

65. Wilkinson IB, Franklin SS, Hall IR, et al. (2001) Pressure amplification explains why pulse pressure is unrelated to risk in young subjects. Hypertension 38, 1461-1466.

66. Galisteo M, Duarte J \& Zarzuelo A (2008) Effects of dietary fibers on disturbances clustered in the metabolic syndrome. J Nutr Biochem 19, 71-84.

67. Reeves RA (1991) A review of the stability of ambulatory blood pressure: implications for diagnosis of hypertension. Clin Invest Med 14, 251-255.

68. Brown DE, James GD \& Nordloh L (1998) Comparison of factors affecting daily variation of blood pressure in Filipino-American and Caucasian nurses in Hawaii. Am J Phys Anthropol 106, 373-383.

69. Jenkins DJ, Kendall CW, Vuksan V, et al. (2002) Soluble fiber intake at a dose approved by the US Food and Drug Administration for a claim of health benefits: serum lipid risk factors for cardiovascular disease assessed in a randomized controlled crossover trial. Am J Clin Nutr $\mathbf{7 5}$, 834-839.

70. Harriss LR, English DR, Powles J, et al. (2007) Dietary patterns and cardiovascular mortality in the Melbourne Collaborative Cohort Study. Am J Clin Nutr 86, 221-229.

71. Coudray CDC \& Rayssiguier Y (2003) Effects of dietary fibers on magnesium absorption in animals and humans. $J$ Nutr 133, $1-4$.

72. Greger J (1999) Nondigestible carbohydrates and mineral bioavailability. J Nutr 129, 1434S-1435S.

73. Bessesen D (2001) The role of carbohydrates in insulin resistance. J Nutr 131, 2782S-2786S.

74. Ferrannini E, Haffner SM, Mitchell BD, et al. (1991) Hyperinsulinaemia: the key feature of a cardiovascular and metabolic syndrome. Diabetologia 34, 416-422. 
75. King DE, Mainous AG III, Egan BM, et al. (2005) Fiber and C-reactive protein in diabetes, hypertension, and obesity. Diabetes Care 28, 1487-1489.

76. Qi L, Rimm E, Liu S, et al. (2005) Dietary glycemic index, glycemic load, cereal fiber, and plasma adiponectin concentration in diabetic men. Diabetes Care 28, 1022-1028.

77. Henry RM, Kostense PJ, Spijkerman AM, et al. (2003) Arterial stiffness increases with deteriorating glucose tolerance status: the Hoorn Study. Circulation 107, 2089-2095.

78. Schram MT, Henry RM, van Dijk RA, et al. (2004) Increased central artery stiffness in impaired glucose metabolism and type 2 diabetes: the Hoorn Study. Hypertension 43, $176-181$.

79. Seals DR, Moreau KL, Gates PE, et al. (2006) Modulatory influences on ageing of the vasculature in healthy humans. Exp Gerontol 41, 501-507.

80. Brock DW, Davis CK, Irving BA, et al. (2006) A high-carbohydrate, high-fiber meal improves endothelial function in adults with the metabolic syndrome. Diabetes Care 29, $2313-2315$

81. Wilkinson IB, Qasem A, McEniery CM, et al. (2002) Nitric oxide regulates local arterial distensibility in vivo. Circulation 105, 213-217. 